\title{
Temporal integration shown in the late component of the human blink reflex
}

\author{
GEOFF HAMMOND, PETER DRISCOLL, and KIRK ROWLEY \\ University of Western Australia, Nedlands, Australia
}

\begin{abstract}
The effect of closely spaced stimulus pairs (S1-S2), one at or above threshold for eliciting the second electromyographic burst of the cutaneous blink reflex (R2) and one below threshold intensity, on the latency, duration, and area of R2 was examined in four experiments. The size of the R2 elicited by a threshold S1 (but not by an S1 at three times threshold intensity) was augmented by threshold or nearthreshold S2s that appeared after stimulus onset asynchronies of 4,8 , and $16 \mathrm{msec}$. More intense S2s had a greater effect on R2 size and led also to a slight shortening of R2 latency. Although R2 augmentation was accompanied by a modest increase in response duration, this increase in duration was by itself insufficient to account for the augmentation of response amplitude. Subreflexogenic S1s (at 0.1, $0.2,0.4$, and 0.8 times $\mathrm{R} 2$ threshold intensity) did not affect the R2 elicited by an S2 at threshold intensity or two times threshold intensity. Unlike the simple brainstem pathway which mediates Rl, the more complex brainstem pathway which mediates $\mathrm{R} 2$ is capable of summing stimulus energy over time.
\end{abstract}

The ability of the nervous system to integrate stimulus energy distributed over time, and its opposite, the ability of the nervous system to resolve stimulus energy that is distributed in time, are fundamental properties thought in part to determine higher order psychological functions (see, e.g., Tallal, Galaburda, Llinás, \& von Euler, 1993). These processes have usually been studied with psychophysical procedures which require subjects to make sensory judgments, but they have also been studied with reflex procedures in which features of reflex behavior such as amplitude, latency, probability, and the reflex-elicitation threshold have been used as objective indicators of the temporal features of sensory processing.

Most studies have examined the effects of manipulating the temporal characteristics of the eliciting stimulus (or stimuli) on reflex expression. Marsh, Hoffman, and Stitt (1973) first showed that the amplitude of the acoustic startle reaction in rats was sensitive to the duration of a continuous acoustic eliciting stimulus up to a limiting duration of about $8 \mathrm{msec}$ and to presentation of paired reflexeliciting stimuli up to a limiting interval of about $11 \mathrm{msec}$. They interpreted these results as an instance of temporal summation of stimulus energy in the brainstem mechanism responsible for expression of the startle reaction. The acoustic startle reaction in rats is also augmented by trailing nonreflexogenic acoustic and percutaneous stimuli that appear within about $8 \mathrm{msec}$ of the startle-eliciting stimulus (Hammond \& Leitner, 1990). Generally similar findings to those of Marsh et al. (1973) have been reported for the acoustic blink reflex in adult humans by Blumenthal

Send correspondence to G. Hammond, Department of Psychology, University of Western Australia, Nedlands, WA 6907, Australia (e-mail: geoff@psy.uwa.edu.au).

-Accepted by previous editor, Paul E. Gold and Berg (1986), who reported that reflex amplitude increased with duration of a continuous eliciting noise and with the interval between successive noise bursts to a limiting time of between 35 and $50 \mathrm{msec}$ in both cases. Manipulating the interval between paired stimuli had no effect on latency, whereas latency was shortened by an average of about $4 \mathrm{msec}$ with increased stimulus duration. Like Marsh et al. (1973), Blumenthal and Berg interpreted their results as showing temporal integration of stimulus energy. Comparable results to those in adult humans have been reported for the acoustic blink reflex in neonates (Blumenthal, Avendano, \& Berg, 1987). The finding that the amplitude of the acoustic blink reflex in adult humans is augmented with paired acoustic reflex-eliciting stimuli has been replicated and shown also to be present with paired percutaneous eliciting stimuli (brief shocks to the supraorbital nerve; Plant \& Hammond, 1989). Furthermore, amplitude augmentation was found with cross-modal stimulus pairs (acoustic-percutaneous and percutaneousacoustic). The time course of the decay of augmentation was similar with all stimulus pairings, reaching asymptote at single-stimulus control levels between 36 and $72 \mathrm{msec}$. There was some latency shortening at brief stimulus onset asynchronies (SOAs) in all paired stimulus conditions with the exception of the percutaneous stimulus pairs.

The amplitude of the short-latency R1 component of the cutaneous blink reflex (which is mediated by a simple oligosynaptic brainstem pathway in contrast to the polysynaptic pathway which mediates the longer latency R2) is augmented with successive percutaneous stimuli at SOAs from 1 to $8 \mathrm{msec}$ but not with percutaneous-acoustic stimulus pairs presented at the same SOAs (Hammond \& Dennon, 1991), although the trailing acoustic stimulus increased the amplitude and reduced the latency of the R2 component elicited by the leading percutaneous stimulus. Rl augmentation with percutaneous stimulus pairs in- 
creased with the intensity of the second stimulus and was present when the second stimulus was itself below threshold for reliable elicitation of R1. A second series of experiments (Hammond \& Plant, 1993) showed that percutaneous stimulus pairs ( $\mathrm{S} 1-\mathrm{S} 2)$ separated by SOAs as brief as $4 \mathrm{msec}$ elicited separate successive Rl components, not a single compound component. The R1 component elicited by the $\mathrm{S} 1$ was unaffected by an S2 presented at SOAs from 4 to $16 \mathrm{msec}$. However, the frequency with which the S2 elicited an R1, and its size, were both increased by the presence of an S1. Augmentation of the $\mathrm{R} 1$ elicited by the $\mathrm{S} 2$ was a smoothly decreasing function of the SOA, reaching an asymptote at $12-15 \mathrm{msec}$. These results cannot be explained in terms of temporal integration of stimulus energy but show instead a "priming" effect in that a percutaneous stimulus evokes a transient excitation of the R1 pathway which augments the amplitude of an R1 elicited by a second percutaneous stimulus presented before the excitation has decayed.

Augmentation of R2 with S1-S2 stimulus pairs is well established, but the mechanism is not known. R2 augmentation may be at least partly due to priming (the mechanism of R1 augmentation), or it may be that the R2 pathway, which is more complex than the Rl pathway, allows true temporal integration in that the energy of a trailing stimulus summates with that of a leading reflex-eliciting stimulus and increases its behavioral impact.

The general aim of the present experiments was to examine changes in the area, duration, and latency of the R2 component of the cutaneous blink reflex with closely spaced stimulus pairs to determine whether augmentation is due to temporal summation of stimulus energy, a priming effect of the leading stimulus, or both.

\section{EXPERIMENT 1}

The aim of Experiment 1 was to determine changes in R2 latency, duration, and area when a suprathreshold S1 was followed by an S2 which was either reflexogenic (with its intensity set at the R2 elicitation threshold) or subreflexogenic (with its intensity at half threshold intensity). It was of particular interest to determine whether R2 augmentation, if present, was a result of increased response duration, which would implicate elicitation of separate successive $\mathrm{R} 2$ responses as the mechanism responsible.

\section{Method}

Subjects. Six volunteers ( 5 males) whose ages ranged from 20 to 23 years of age were tested. All subjects in this and later experiments gave informed consent to the procedures which had been approved by the University's Committee for Human Rights.

Apparatus and Procedure. All testing was done with the subjects seated comfortably with eyes open in a dimly lit soundattenuating room. Blink reflexes were elicited by current-controlled 0.2 -msec square-wave pulses applied through gold-plated electrodes attached over the supraorbital branch of the trigeminal nerve. The EMG activity of the orbicularis oculi muscle was recorded with two 6-mm-diameter gold-plated electrodes, one placed over the palpebral portion of the muscle and the other at the lateral canthus; a similar ground electrode was placed on the mastoid. The EMG was amplified by a Grass amplifier with half-amplitude high- and low-pass frequencies at $1 \mathrm{~Hz}$ and $1 \mathrm{kHz}$, respectively.

The threshold current to elicit four successive R2 responses was determined by a single ascending method of limits. Each subject was then given five trials in each of the eight $\mathrm{S} 1-\mathrm{S} 2$ conditions defined by the factorial combination of four SOAs $(4,8,16$, and $32 \mathrm{msec}$ ) and two S2 intensities (the R2 elicitation threshold and 0.5 times the R2 threshold; $1 \mathrm{~T}$ and $0.5 \mathrm{~T}$, respectively) and $10 \mathrm{~S} 1$-alone trials for a total of 50 trials. S1 intensity was always at three times the R2 elicitation threshold (3T). One trial of each of the eight S1-S2 conditions and two S1-alone trials (to establish control levels of reflex activity) were given in random blocks of 10 trials.

Data analysis. The amplified and filtered EMG was subject to 12-bit analog-to-digital conversion at $4 \mathrm{kHz}$ for $150 \mathrm{msec}$ beginning with the onset of $\mathrm{S} 1$. The values were saved to disk and later analyzed with locally written software which recreated the EMG trace on a computer screen and allowed scoring of the onset and offset latencies and the area of the $\mathrm{R} 2$ burst. In each block of trials, the area of the R2 elicited in each of the eight paired stimulus conditions was expressed as a ratio of the mean area of the two R2s elicited on the single-stimulus control trials within that trial block. Medians of these amplitude ratios were used for later analysis to eliminate the effect of infrequent extreme ratio scores. Means of each subject's median R2 latency and duration (in milliseconds) in each condition were used for later analysis. Confidence intervals around these means were calculated following the method suggested by Loftus and Masson (1994). This method ignores betweensubjects variability in the parameter under investigation in withinsubjects designs when its absolute value is of no interest, thereby allowing more precise estimates of change in the parameter with experimental conditions.

\section{Results and Discussion}

In 2 subjects, the $\mathrm{R} 2$ elicited by $\mathrm{S} 1$ was contaminated by an $\mathrm{S} 2$-elicited R1 on some trials with the $32-\mathrm{msec} \mathrm{SOA}$. These responses occurred presumably because the $S 1$ increased the excitability of the blink pathway sufficiently for the S2 to elicit an R1 although it was by itself below $\mathrm{R} 1$ threshold. The possibility of similar contamination on other trials in these subjects (and possibly in other subjects) without being evident led us to drop all $32-\mathrm{msec}$ SOA trials from the analysis. There was no evidence of amplitude augmentation in the remaining S1-S2 conditions, with all mean amplitude ratios close to one (Table 1). R2 duration was also unaffected by the experimental conditions. The mean median $\mathrm{R} 2$ duration in the singlestimulus control condition was $36 \mathrm{msec}$, with a $95 \%$ confidence interval from 33 to $40 \mathrm{msec}$; 2 durations in the paired-stimulus conditions ranged from 37 to $39 \mathrm{msec}$. Similarly, R2 latency was unaffected by the experimen-

Table 1

Mean Median Amplitude Ratios at Each Stimulus Onset Asynchrony (SOA, in Milliseconds) for the Low-Intensity S2

(0.5 Times R2 Threshold Intensity) and High-Intensity S2 (1.0 Times R2 Threshold Intensity) in Experiment 1

\begin{tabular}{lccc}
\hline & \multicolumn{3}{c}{ SOA } \\
\cline { 2 - 4 } & 4 & 8 & 16 \\
\hline Low-intensity S2 & 0.91 & 1.12 & 1.01 \\
& $(0.54-1.28)$ & $(0.75-1.49)$ & $(0.64-1.38)$ \\
High-intensity S2 & 1.16 & 0.91 & 1.15 \\
& $(0.79-1.53)$ & $(0.54-1.28)$ & $(0.78-1.52)$
\end{tabular}

Note-The $95 \%$ confidence intervals are in parentheses. 
tal conditions. The mean median R2 latency was $38 \mathrm{msec}$ $(95 \%$ confidence interval $=36-40 \mathrm{msec})$ in the control condition, and it ranged from 36 to $40 \mathrm{msec}$ in the pairedstimulus conditions. The absence of augmentation in the present experiment was unexpected, and it could have resulted from a ceiling effect: S1 intensity, at three times the R2 elicitation threshold, may have elicited near-maximal $\mathrm{R} 2 \mathrm{~s}$, thereby preventing augmentation.

\section{EXPERIMENT 2}

This experiment repeated the conditions of the first experiment with the exception that a lower S1 intensity (IT) was used.

\section{Method \\ Subjects. Six volunteers ( 4 males) whose ages ranged from 19 to 22 years were tested. Three had participated in Experiment 1. \\ Apparatus and Procedure. The apparatus and general proce- dure were the same as those in Experiment 1. The experimental con- ditions were identical to those in Experiment 1 with the exception that the intensity of S1 was set at the R2 elicitation threshold (1T) for each subject.}

\section{Results and Discussion}

Analysis was again restricted to the 4-, 8-, and 16-msec SOAs. Means of the median R2 amplitude ratios for both S2 intensities at each of the three SOAs are in Table 2. Amplitude augmentation was present with the more intense S2 (1T), and it declined systematically with SOA. No amplitude augmentation was present with the less intense S2 $(0.5 \mathrm{~T})$, where all ratios were close to one. These results are consistent with previous observations which have shown that the size of the R2 component of the cutaneous blink reflex and the single component of the acoustic blink reflex (which appears to be functionally equivalent to the cutaneous R2; Hackley \& Boelhouwer, in press) is augmented when a reflex-eliciting $\mathrm{S} 1$ is followed by a reflex-eliciting $\mathrm{S} 2$. The mean median durations of the augmented $\mathrm{R} 2 \mathrm{~s}$ elicited with the more intense S2 at the 4- and 8-msec SOAs ( 34 and $32 \mathrm{msec}$, respectively) were both longer than the mean median duration of the control responses $(26 \mathrm{msec} ; 95 \%$ confidence interval $=21-31 \mathrm{msec}$ ). These increases in response duration (about $31 \%$ and $23 \%$ respectively) are not by themselves sufficient to account for the doubling of response area in these conditions. Augmentation of R2

Table 2

Mean Median Amplitude Ratios at Each Stimulus Onset Asynchrony (SOA, in Milliseconds) for the Low-Intensity S2 (0.5 Times R2 Threshold Intensity) and High-Intensity S2 (1.0 Times R2 Threshold Intensity) in Experiment 2

\begin{tabular}{lccc}
\hline & \multicolumn{3}{c}{ SOA } \\
\cline { 2 - 4 } & 4 & 8 & 16 \\
\hline Low-intensity S2 & 0.99 & 0.91 & 0.81 \\
& $(0.51-1.47)$ & $(0.43-1.39)$ & $(0.33-1.29)$ \\
High-intensity S2 & 2.56 & 1.97 & 1.29 \\
& $(2.08-3.04)$ & $(1.49-2.45)$ & $(0.81-1.77)$ \\
\hline
\end{tabular}

Note-The $95 \%$ confidence intervals are in parentheses. amplitude with paired stimuli in these conditions is therefore at least in part a result of integration of the energy of the two stimuli, leading to a larger response to the first stimulus in the pair. The longer response durations found with the higher intensity S2 at the 4- and 8-msec SOAs might be part of integration-integrated responses might be longer as well as being of greater size--or might reveal the presence of a response to $\mathrm{S} 2$ which merges with that elicited by S1. Two observations favor the former interpretation: first, the duration of the $\mathrm{R} 2$ elicited by the higher intensity S2 at the 16 -msec SOA $(28 \mathrm{msec})$ was not appreciably longer than that of the control responses; and second, if $\mathrm{S} 2$ had elicited a second R2 which merged with that elicited by S1, R2 duration would have increased progressively with SOA as the second R2 was elicited progressively later. There was no systematic variation of R2 latency in the different conditions. The mean median control latency was $44 \mathrm{msec}(95 \%$ confidence interval $=41-47 \mathrm{msec}$ ), and in the paired-stimulus conditions, latencies ranged from 43 to $46 \mathrm{msec}$.

\section{EXPERIMENT 3}

The previous experiment showed that R2 augmentation with paired stimuli was dependent on S2 intensity, and that although powerful with S2 at 1T, it was negligible with S2 at $0.5 \mathrm{~T}$. The purpose of Experiment 3 was to see whether the finding of Experiment 2, that R2 amplitude augmentation with threshold-level stimulus pairs occurs without response prolongation, would be replicable and to determine whether augmentation of R2 would be present with a subreflexogenic S2 at a higher intensity than that in Experiment 2.

\section{Method}

Subjects. Twelve volunteers ( 7 males) whose ages ranged from 19 to 51 years (median $=22$ years) were tested. Four had participated in at least one of the two previous experiments.

Apparatus and Procedure. The apparatus and general procedure were the same as those used in the previous experiments. The experimental conditions were identical to those in Experiment 2, with the exception that the intensity of the low-intensity S2 was set at $80 \%$ of the R2 elicitation threshold $(0.8 \mathrm{~T})$ for each subject.

\section{Results and Discussion}

Mean median amplitude ratios are in Table 3. Augmentation of R2 area was present for both S2 intensities, $0.8 \mathrm{~T}$ and $1 \mathrm{~T}$, but was greater with the more intense $\mathrm{S} 2$. Response durations were again longer in the paired-stimulus conditions than in the control condition. The mean median duration of the control responses was $27 \mathrm{msec}(95 \%$ confidence interval $=25-29 \mathrm{msec}$ ). With the low-intensity S2, mean median response durations at the 4-, 8-, and 16-msec SOAs were 32,30 , and $31 \mathrm{msec}$, respectively; with the high-intensity $\mathrm{S} 2$, the corresponding values were 35,32 , and $31 \mathrm{msec}$, respectively. As in the previous experiment, the prolongation of $\mathrm{R} 2$ was insufficient by itself to account for the observed increase in amplitude. For example, the more intense S2 at the 4-msec SOA more than trebled $\mathrm{R} 2$ amplitude relative to control, yet duration in- 
Table 3

Mean Median Amplitude Ratios at Each Stimulus Onset Asynchrony (SOA, in Milliseconds) for the Low-Intensity S2 (0.8 Times R2 Threshold Intensity) and High-Intensity $\mathrm{S} 2$ (1.0 Times R2 Threshold Intensity) in Experiment 3

\begin{tabular}{|c|c|c|c|}
\hline & \multicolumn{3}{|c|}{ SOA } \\
\hline & 4 & 8 & 16 \\
\hline Low-intensity S2 & $\begin{array}{c}2.50 \\
(1.72-3.28)\end{array}$ & $\begin{array}{c}2.11 \\
(1.33-2.89)\end{array}$ & $\begin{array}{c}1.71 \\
(0.93-2.49)\end{array}$ \\
\hline High-intensity S2 & $\begin{array}{c}3.50 \\
(2.72-4.28)\end{array}$ & $\begin{array}{c}3.08 \\
(2.30-3.86)\end{array}$ & $\begin{array}{c}2.24 \\
(1.46-3.02)\end{array}$ \\
\hline
\end{tabular}

Note-The $95 \%$ confidence intervals are in parentheses.

creased by only about $30 \%$. A similar disproportionality of increases in amplitude and duration was evident in the other conditions. Furthermore, response duration did not increase with SOA by an amount equal to the SOA in the way that would be expected if the second stimulus had elicited a second R2 which extended the apparent response duration. Mean median latency was $46 \mathrm{msec}(95 \%$ confidence interval $=45-47 \mathrm{msec}$ ) in the single-stimulus control condition. There was a small facilitation of the response in the paired-stimulus conditions with the high-intensity S2 in which the mean median latencies ranged from 43 to $44 \mathrm{msec}$. Latencies in the paired-stimulus conditions with the low-intensity $\mathrm{S} 2$ ranged from 45 to $47 \mathrm{msec}$.

These observations confirm the general finding of Experiment 2 that $\mathrm{R} 2$ amplitude is augmented when a reflexeliciting stimulus is followed within $16 \mathrm{msec}$ by a second reflex-eliciting stimulus. Pooling the data from the pairedstimulus conditions with the higher intensity S2 from Experiments 2 and 3 yielded mean median amplitude ratios (with the $95 \%$ confidence limits in parentheses) of $3.37(2.62-4.12), 2.85(2.10-3.60)$, and $2.00(1.25-2.75)$ for the 4-, 8-, and 16-msec SOAs, respectively. It is clear in both Experiments 2 and 3 that although this augmentation is accompanied by an increase in $\mathrm{R} 2$ duration, the increased duration is not sufficient for the augmentation to be merely the result of an $\mathrm{R} 2$ to the trailing stimulus. These observations extend those of Experiment 2 by showing that $\mathrm{R} 2$ augmentation is greater with a more intense $\mathrm{S} 2$, that $\mathrm{S} 2$ need not itself be reflexogenic in order to augment the response to $S 1$, and that augmentation can be accompanied by a small latency reduction.

\section{EXPERIMENT 4}

The previous experiments illustrate that $\mathrm{R} 2$ is augmented when a second stimulus follows an initial reflexeliciting stimulus at threshold intensity but not at three times threshold intensity. Augmentation depends on S2 intensity and is present when $S 2$ is reflexogenic or nearreflexogenic, but not when its intensity is half that of the $\mathrm{R} 2$ elicitation threshold. Some increase in response duration accompanies augmentation in these conditions, but it is not sufficient to imply that the mechanism of augmentation is merely the addition of a second $R 2$ elicited by the S2. Unlike R1, one mechanism of augmentation for $\mathrm{R} 2$ with paired stimuli is temporal summation of the stimulus energy. The aim of Experiment 4 was to determine whether priming is also a mechanism of R2 augmentation with paired stimuli-that is, to determine whether R2 is augmented when a nonreflexogenic S1 precedes a reflexogenic $\mathrm{S} 2$ by a brief interval.

\section{Method}

Subjects. Nine volunteers ( 5 males) whose ages ranged from 19 to 50 years (median $=23$ years) were tested. Two subjects had participated in one previous experiment, and 1 had participated in two previous experiments.

Apparatus and Procedure. The apparatus and general procedure were the same as those in the previous experiments. Each subject was given 10 trials in each of the eight paired-stimulus (S1-S2) conditions defined by the factorial combination of four $\mathrm{S} 1$ intensities $(0.1,0.2,0.4$, and 0.8 times $\mathrm{R} 2$ elicitation threshold) and two S2 intensities ( 1 and 2 times R2 threshold). Ten single-stimulus trials at each S2 intensity were given for a total of 100 trials. S1 and S2 were separated by $8 \mathrm{msec}$ on paired-stimulus trials. One trial of each of the eight paired-stimulus conditions and one trial at each of the singlestimulus intensities were given in random blocks of 10 trials.

\section{Results and Discussion}

Mean median amplitude ratios are in Table 4 . These data show some augmentation of R2 area with the most intense S1 (0.8T) paired with the less intense S2 (1T). All other amplitude ratios were close to 1 , showing no appreciable effect of the leading subreflexogenic stimuli on $\mathrm{R} 2$ area. There was no effect of the experimental conditions on R2 duration. The mean median duration of the R2 elicited by the low-intensity S2 (1T) given alone was $36 \mathrm{msec}(95 \%$ confidence interval $=32-40 \mathrm{msec})$ while the mean median durations in the paired-stimulus conditions with this $\mathrm{S} 2$ ranged from 34 to $38 \mathrm{msec}$; the mean median duration of the $\mathrm{R} 2$ elicited by the high-intensity S2 (2T) given alone was $41 \mathrm{msec}(95 \%$ confidence inter$\mathrm{val}=37-45 \mathrm{msec}$ ) while the mean median durations in the paired-stimulus conditions with this $\mathrm{S} 2$ ranged from 44 to 45 msec.

R2 latency, in contrast to duration, did vary with experimental condition (see Table 5). First, as expected, the more intense eliciting stimulus evoked R2s with shorter latency than did the less intense stimulus. The difference in the control S2-alone conditions was about $5 \mathrm{msec}$. Of more interest is an abrupt shortening of R2 latency in the paired-stimulus conditions with the most intense $\mathrm{Sl}$; as was the case with R2 area, this change was more pro-

Table 4

Mean Median Amplitude Ratios at Each S1 Intensity (0.1, 0.2, 0.4, and 0.8 Times R2 Threshold Intensity) and Each S2 Intensity (1.0 and 2.0 Times R2 Threshold Intensity) in Experiment 4

\begin{tabular}{lcccc}
\hline & \multicolumn{4}{c}{ S1 Intensity (re Threshold) } \\
\cline { 2 - 5 } & $0.1 \mathrm{~T}$ & $0.2 \mathrm{~T}$ & $0.4 \mathrm{~T}$ & $0.8 \mathrm{~T}$ \\
\hline Low-intensity S2 & 0.86 & 0.92 & 0.92 & 1.40 \\
& $(0.63-1.09)$ & $(0.69-1.15)$ & $(0.69-1.15)$ & $(1.17-1.63)$ \\
High-intensity S2 & 0.99 & 1.12 & 1.01 & 1.11 \\
& $(0.76-1.22)$ & $(0.89-1.35)$ & $(0.78-1.24)$ & $(0.88-1.34)$ \\
\hline
\end{tabular}

Note-The $95 \%$ confidence intervals are in parentheses. 
Table 5

Mean Median R2 Latency (in Milliseconds) at Each S1 Intensity $(0.1,0.2,0.4$, and 0.8 Times $\mathrm{R} 2$ Threshold Intensity) and Each S2 Intensity (1.0 and 2.0 Times R2 Threshold Intensity) in Experiment 4

\begin{tabular}{lccccc} 
& & \multicolumn{4}{c}{ S1 Intensity (re Threshold) } \\
\cline { 3 - 6 } & S2 alone & $0.1 \mathrm{~T}$ & $0.2 \mathrm{~T}$ & $0.4 \mathrm{~T}$ & $0.8 \mathrm{~T}$ \\
\hline Low-intensity S2 & 44 & 44 & 43 & 43 & 36 \\
& $(42-46)$ & $(42-46)$ & $(41-45)$ & $(41-45)$ & $(34-38)$ \\
High-intensity S2 & 39 & 39 & 38 & 40 & 36 \\
& $(37-41)$ & $(37-41)$ & $(36-40)$ & $(38-42)$ & $(34-38)$ \\
\hline
\end{tabular}

Note-The $95 \%$ confidence intervals are in parentheses.

nounced with the less intense $\mathrm{S} 2$. The shortening of average latency suggests that the $\mathrm{R} 2$ was elicited, on at least some trials, by S1 rather than by the nominal eliciting stimulus, S2. Such cases, if they occurred, could potentially account for the augmentation of R2 area noted in these conditions: rather than S1 acting to augment the subsequently elicited R2, it might have been that augmentation of response area occurred when, owing to uncontrolled fluctuations in the excitability of the reflex pathway, a normally subreflexogenic Sl elicited a blink and was followed $8 \mathrm{msec}$ later by an S2 which was set at the previously determined reflex-elicitation threshold. This possibility was investigated for the less intense $\mathrm{S} 2$ by calculating the $95 \%$ confidence limits for the mean control latency (i.e., the R2 latency on the 1T single-stimulus trials) for each subject. R2s elicited in the $0.8 \mathrm{~T}-1 \mathrm{~T}$ pairedstimulus condition whose latency was shorter than the lower confidence limit of the latency of the 1T control trials were identified as probable responses to the leading stimulus, the $0.8 \mathrm{~T} \mathrm{S1}$. On the average, $58 \%$ of trials in the $0.8 \mathrm{~T}-1 \mathrm{~T}$ condition met this criterion and were judged to be responses to $\mathrm{Sl}$ (the percentages ranged from $0 \%$ to $100 \%$ in the individual subjects). The average amplitude ratio of the short-latency responses identified in the $0.8 \mathrm{~T}-1 \mathrm{~T}$ condition was 1.51 , whereas it was .80 for the responses whose latency was longer than the lower $95 \%$ confidence limit of the mean latency on the $1 \mathrm{~T}$ control trials and which were therefore taken as probable responses to the S2 (these amplitude ratios are each means of values from 8 subjects; 1 subject had no "fast" responses, and 1 subject showed all "fast" responses). The average amplitude ratio of 1.51 for the R2s putatively evoked by $\mathrm{S} 1$ is comparable to that of 1.97 obtained with $1 \mathrm{~T}-1 \mathrm{~T}$ stimulus pairs separated by $8 \mathrm{msec}$ in Experiment 2 . This analysis could not be done for the $0.8 \mathrm{~T}-2 \mathrm{~T}$ condition, because the shorter latency of the R2s elicited by the more intense $\mathrm{S} 2$ (i.e., the $2 \mathrm{~T}$ eliciting stimulus) could not be differentiated from the slower R2s elicited by the $8-\mathrm{msec}$ earlier but just reflexogenic $\mathrm{S}$. The two latency distributions-one of the R2s to the S2 set at 2T and one potentially from the $\mathrm{S} 1$ set at $0.8 \mathrm{~T}$ - would overlap extensively as the 8 -msec lead to an S1-elicited R2 would be offset by the shortened latency to the more intense $\mathrm{S} 2$ (the mean R2 latency difference between responses to $1 \mathrm{~T}$ and $2 \mathrm{~T}$ control stimuli was about $5 \mathrm{msec}$ ). These results, together with the absence of augmentation with the other leading stimuli (.1T, .2T, and .4T) suggest quite strongly that R2, unlike $\mathrm{R} 1$, is not augmented by subreflexogenic leading stimuli.

\section{GENERAL DISCUSSION}

The general issue addressed by this and several previous reports is how modification of the blink reflex with closely spaced stimulus pairs can be used to measure how the nervous system integrates and resolves stimuli that are close together in time. The two successive components of the cutaneous blink reflex give it the potential of measuring these processes separately in a simple and in a more complex brainstem pathway. The general results of these experiments are straightforward. $\mathrm{R} 1$, which is mediated by a simple pathway, shows a priming effect (the presence of $\mathrm{S} 1$ augments the R1 elicited by a subsequent S2) but does not show temporal integration (the presence of an S2 does not affect the size of the R1 elicited by an $\mathrm{S} 1$ ). R2, which is mediated by a more complex pathway, does not show priming (an S1 does not affect the size of the R2 elicited by an S2) but does show temporal integration (an S2 augments the R2 elicited by an S1 without any sign that the $\mathrm{S} 2$ itself elicits a response).

Priming by a percutaneous $\mathrm{S} 1$, which can be studied in $\mathrm{R} 1$, shows a substantial increase in excitability of the $\mathrm{R} 1$ pathway (increases of $500 \%$ are common) that decays over a period of $12-15 \mathrm{msec}$. Priming from a percutaneous $\mathrm{S} 1$ occurs when it is subreflexogenic $(0.8 \mathrm{~T})$ and there is little if any effect of intensity above threshold, at least over a limited range of intensities from 1T to 1.4T (Hammond \& Plant, 1993). Priming increases with S2 intensity, showing that more intense S2s are more able to exploit the transient increase in the excitability of the R 1 pathway triggered by S1 (Hammond \& Plant, 1993). R1 amplitude is augmented by an acoustic $\mathrm{S} 1$, showing that priming occurs with $\mathrm{S} 1$ and $\mathrm{S} 2$ in different sensory modalities (Boelhouwer, Teurlings, \& Brunia, 1991). In Boelhouwer et al.'s experiment, augmentation reached an average maximum of about $700 \%$ of control amplitude and was present at SOAs from about 10 to $100 \mathrm{msec}$, revealing a more prolonged time course than for priming of $\mathrm{R} 1$ by a percutaneous $\mathrm{S} 1$. The different time courses might reflect the relatively long $\mathrm{Sl}$ duration used by Boelhouwer et al. ( $50 \mathrm{msec}$, as opposed to $0.2 \mathrm{msec}$ in studies of priming by a percutaneous $\mathrm{S} 1$ ) or perhaps genuine modality differences, with acoustic stimuli exerting a more sustained excitatory effect than that of percutaneous stimuli. Other variables, such as $\mathrm{S} 1$ repetition, have not been studied. If modifiable, priming may be an elementary form of neural plasticity with some use as an easily studied model of neural plasticity in humans.

Temporal integration, which can be studied in $\mathrm{R} 2$, is the incorporation of the energy of a trailing stimulus into the response elicited by a leading stimulus. Reflex expression is not determined completely by the stimulus features present in the onset of the eliciting stimulus, but events that occur after the initiating stimulus in the period of the reflex latency add to reflex size and can also 
shorten its onset latency. The occurrence of temporal integration for R2 but not R1 implies that it occurs in the central pathways of $\mathrm{R} 2$ which are not shared with $\mathrm{R} 1$. This phenomenon has been studied quite extensively with the use of both the human blink reflex and the acoustic startle reaction in rats as the response indicator (Blumenthal \& Berg, 1986; Marsh et al., 1973; Plant \& Hammond, 1989). The pooling of energy is time dependent: response augmentation is greatest at brief stimulus separations and declines systematically as the temporal separation is increased. Substantial augmentation is obtained-increases of about $200 \%-300 \%$ are typicalbut it is not usually as great as augmentation due to priming in $\mathrm{R} 1$. Temporal integration occurs cross-modallyacoustic and percutaneous $S 2$ s augment the R2s elicited by percutaneous and acoustic S Is respectively-and is intensity dependent with greater augmentation from more intense S2s. Although this reflex phenomenon is robust, its comparison to temporal integration of stimulus energy studied psychophysically is unconvincing. First, temporal integration shown by $\mathrm{R} 2$ occurs with $\mathrm{S} 1$ and $\mathrm{S} 2$ in different sensory modalities, and its time course is reflex- (not modality-) dependent, being brief for the shortlatency acoustic startle reaction in rats (Marsh et al., 1973) and longer for the long-latency human blink reflex (Blumenthal \& Berg, 1986; Plant \& Hammond, 1989). Second, temporal integration of acoustic and percutaneous stimuli measured by augmentation of $\mathrm{R} 2$ has a much longer time course than that found for integration of these stimuli measured psychophysically, where integration appears to be limited to less than $5 \mathrm{msec}$ for threshold acoustic pulses (Viemeister \& Wakefield, 1991) and to less than about $1 \mathrm{msec}$ for threshold percutaneous stimuli (Higashiyama \& Tashiro, 1983, 1988; Rollman, 1969) and about 5-7 msec for suprathreshold percutaneous stimuli (Higashiyama \& Tashiro, 1988; Uttal, 1959). In contrast, R2 augmentation is found with separations up to about 50 msec (Blumenthal \& Berg, 1986). Temporal integration shown in reflex behavior appears to reflect a summation process that is specific to reflex expression rather than sensory processing.

The occurrence of priming for R1 but not R2 may simply reflect the different latencies of these two EMG components: the neural processes which lead to R1 occur quickly enough after the eliciting S2 to exploit the transient wave of excitation evoked by $\mathrm{S} 1$, whereas the processes that lead to R2 may not occur until after the excitation has decayed. The occurrence of temporal integration for R2 but not R1 may reflect the different properties of the pathways of the two components. Temporal integration may depend on processes of sufficient duration that occur in the complex R2 pathway but not in the simple $\mathrm{R} 1$ pathway. The expression of $\mathrm{R} 2$ is not ballistic, but is the result of processes which, because of their duration, come with a window of sensitivity during which appropriate stimuli can augment reflex size and shorten latency. Perhaps this capacity to pool energy across time is functional for defensive reflexes.
Reflex modification procedures offer another means of studying resolution and integration of stimulus energy by manipulating the temporal properties of preliminary stimuli which modify reflex expression. For example, Ison and Pinckney (1983) have shown that a brief interruption of otherwise continuous white noise at an appropriate interval before a reflex-eliciting stimulus inhibits the size of the blink reflex in humans. The threshold gap duration for reflex inhibition (about $5 \mathrm{msec}$ ) corresponds closely with psychophysical estimates. An effect of this nature, unlike reflex modification with stimuli in close temporal contiguity with the reflex-eliciting stimulus which therefore is a consequence only of intrinsic brainstem processes, depends presumably on remote effects acting on the brainstem reflex pathways. The loss of gapinduced inhibition of the rat's startle reaction by cortical spreading depression suggests (at least in this case) activation by the gap of descending inhibitory control of brainstem excitability from the cortex (Ison, O'Connor, Bowen, \& Bocimea, 1991). In a similar vein, Dykman and Ison (1979) showed that inhibition of the startle reaction in rats and the blink reflex in humans increased with the duration of the acoustic prestimulus, suggesting summation of the acoustic energy. Their experimental design allowed the intervals from S1 onset and offset to the eliciting stimulus to covary with S1 duration. More recently, Blumenthal (1995) has reported experiments in which either the interval from $\mathrm{S} 1$ onset to the eliciting stimulus or the interval from $\mathrm{SI}$ offset to the eliciting stimulus was kept constant. Taken together, these experiments (like Dykman \& Ison, 1979) showed increased inhibition of the blink reflex in humans with increased S1 duration, at least to about 20-50 msec. Manipulation of the temporal characteristics of prestimulation in this way appears to be a promising means of studying the temporal characteristics of sensory processing, whereas the procedures used in the present experiments provide an approach to studying the temporal characteristics of motor processing.

\section{REFERENCES}

Blumenthal, T. D. (1995). Prepulse inhibition of the startle eyeblink as an indicator of temporal summation. Perception \& Psychophysics, 57, 487-494.

Blumenthal, T. D., Avendano, A., \& Berg, W. K. (1987). The startle response and auditory temporal summation in neonates. Journal of Experimental Child Psychology, 44, 64-79.

Blumenthal, T. D., \& BerG, W. K. (1986). The startle response as an indicator of temporal summation. Perception \& Psychophysics, $\mathbf{4 0}$, 62-68.

Boelhouwer, A. J. W., Teurlings, R. J. M. A., \& Brunia, C. H. M. (1991). The effect of an acoustic warning stimulus upon the electrically elicited blink reflex in humans. Psychophysiology, 28, 133-139.

Dykman, B. M., \& Ison, J. R. (1979). Temporal integration of acoustic stimulation obtained in reflex inhibition in rats and humans. Journal of Comparative \& Physiological Psychology, 93, 939-945.

HACKLEY, S. A., \& Boelhouwer, A. J. W. (in press). The more or less startling effects of prestimulation-revisited: Prepulse modification of multicomponent blink reflexes. In P. J. Lang. R. F. Simons, \& M. T. Balaban (Eds.), Attention and orienting: Sensory and motivational processes. Hillsdale, NJ: Erlbaum.

Hammond, G. R., \& Dennon, S. (1991). Temporal integration shown 
in the early and late components of the human blink reflex. Psychobiology, 19, 180-186.

Hammond, G. R., \& Leitner, D. S. (1990). Augmentation of the rat's acoustic startle reflex by nonreflexogenic stimuli. Behavioral Neuroscience, 104, 841-848.

Hammond, G. [R.], \& Plant, Y. (1993). Augmentation of the early component of the human blink reflex with closely spaced stimulus pairs. Psychobiology, 21, 69-76.

Higashiyama, A., \& Tashiro, T. (1983). Temporal and spatial integration for electrocutaneous stimulation. Perception \& Psychophysics, 33, 437-442.

HigashiYama, A., \& TASHiro, T. (1988). Temporal integration of double electrical pulses. Perception \& Psychophysics, 43, 172-178.

Ison, J. R., \& Pinckney, L. A. (1983). Reflex inhibition in humans: Sensitivity to brief silent periods in white noise. Perception \& Psychophysics, 34, 84-88.

Ison, J. R., O'Connor, K., Bowen, G. F., \& Bocirnea, A. (1991). Temporal resolution of gaps in noise by the rat is lost with functional decortication. Behavioral Neuroscience, 105, 33-40.

LofTUS, G. R., \& MAsson, M. E. J. (1994). Using confidence intervals in within-subject designs. Psychonomic Bulletin \& Review, 1, 476-490.

MarSh, R., Hoffman, H. S., \& StitT, C. L. (1973). Temporal integra- tion in the acoustic startle reflex of the rat. Journal of Comparative \& Physiological Psychology, 82, 507-511.

Plant, Y., \& Hammond, G. R. (1989). Temporal integration of acoustic and cutaneous stimuli shown in the blink reflex. Perception \& Psychophysics, 45, 258-264.

Rollman, G. B. (1969). Electrocutaneous stimulation: Psychometric functions and temporal integration. Perception \& Psychophysics, 5 , 289-293.

Tallal, P., Galaburda, A. M., Llinás, R. R., \& von Euler, C. (Eds.) (1993). Temporal information processing in the nervous system (Annals of the New York Academy of Sciences, Vol. 682). New York: New York Academy of Sciences.

UTTAL, W. R. (1959). A comparison of neural and psychophysical responses in the somesthetic system. Journal of Comparative \& Physiological Psychology, 52, 485-490.

VIEMEISTER, N. F., \& WAKEFIELD, G. H. (1991). Temporal integration and multiple looks. Journal of the Acoustical Society of America, 90 , 858-865.

(Manuscript received March 27, 1996; revision accepted for publication September 16, 1996.) 